\title{
Projections of local atomic structure revealed by wavelet analysis of x-ray absorption anisotropy
}

\author{
P. Korecki, ${ }^{1, *}$ D. V. Novikov, ${ }^{2}$ and M. Tolkiehn ${ }^{2}$ \\ ${ }^{1}$ Institute of Physics, Jagiellonian University, Reymonta 4, 30-059 Kraków, Poland \\ ${ }^{2}$ HASYLAB at DESY, Notkestraße 85, D-22603 Hamburg, Germany
}

(Received 30 January 2009; revised manuscript received 10 May 2009; published 31 July 2009)

\begin{abstract}
We propose and verify in an experiment a wavelet transform approach for analysis of x-ray absorption anisotropy (XAA) patterns recorded using a broadband polychromatic x-ray beam. XAA results from the interference between an incident plane wave with spherical waves scattered from atoms inside the sample. This interference modifies the total $\mathrm{x}$-ray field amplitude at the sites of absorbing atoms and effectively changes the atomic absorption cross section. XAA is monitored by measuring the secondary yield while the sample is rotated relative to the incident-beam direction. For broadband polychromatic hard x-ray illumination, owing to the short coherence length, significant anisotropy in absorption is only found close to directions of the incident radiation coinciding with interatomic directions. In this work, we show that the signals from individual atoms have the same universal shape and differ only in the scale and angular position. Combined with the directional localization this allows us to construct a spherical wavelet family matched to the shape of the observed signal. Application of the wavelet transform to experimental x-ray absorption anisotropy has provided high-resolution projections of the local atomic structure in an InAs crystal up to the sixth coordination shell. While in a recent work XAA delivered a three-dimensional image of the unit cell obtained through a tomographic algorithm, the wavelet approach provides projections of the local structure of absorber atoms with depth resolution and does not depend on the translational long-range order. This opens a way for a quantitative analysis of polychromatic beam x-ray absorption anisotropy for local structure imaging.
\end{abstract}

DOI: 10.1103/PhysRevB.80.014119

PACS number(s): 61.05.C-, 42.30.-d, 61.05.J-

\section{INTRODUCTION}

X-ray projections have been used for over one hundred years to reveal the internal structure of objects. ${ }^{1}$ Although the depth information is lost in a single radiograph, it is still one of the most frequently used tools for real-space imaging of macroscopic and microscopic objects in medicine, industry, and science. However, obtaining an x-ray projection at the atomic scale is cumbersome. Since the x-ray wavelength is comparable with interatomic distances, wave phenomena are important and $\mathrm{x}$ rays strongly diffract yielding an image in the reciprocal space.

Recently, a qualitative real-space x-ray approach for imaging the atomic structure of solids was proposed., ${ }^{2,3}$ This approach analyzes absorption anisotropy of polychromatic $\mathrm{x}$ rays, which arises due to the interaction between the incident plane wave with spherical waves scattered inside the sample [see Figs. 1(a) and 1(b)]. The scattering geometry changes with the relative orientation of the sample and the direction of the incident beam, which results in variations in the total $\mathrm{x}$-ray field at the sites of absorbing atoms. The anisotropy is monitored by measuring the secondary yield from absorbing atoms while the sample is rotated relative to the incidentbeam direction. Such an experimental geometry is similar to that used for x-ray absorption holography. ${ }^{4-8}$

Holographic methods employ a monochromatic beam and collect information in reciprocal space. They use Fouriertype reconstruction procedures for converting the measured data to the real space. ${ }^{9,10}$ These methods require that the data are recorded over wide angular and energy ranges. If part of the data is corrupted or missing due to experimental factors, the reconstruction procedure will distribute the faulty data over the entire real space, which can yield strong artifacts or deteriorate the spatial resolution. The missing data problem is characteristic of all Fourier-type techniques. It also emerges in other $\mathrm{x}$-ray imaging techniques, e.g., in coherentdiffraction imaging. ${ }^{11,12}$ In order to avoid this problem, holographic data need to be extended to a full sphere, which requires additional a priori information about the sample structure $^{13}$ and is only possible for systems with very high symmetry.

Our approach uses polychromatic x rays to record the absorption anisotropy. ${ }^{2}$ For a broadband polychromatic x-ray beam, a decrease in the longitudinal coherence length causes higher-order diffraction fringes to be extinguished in the $\mathrm{x}$-ray anisotropy. The zero-order diffraction spot, which coincides with the interatomic direction, is energy independent. Thus, for a perfectly white beam, x-ray absorption anisotropy could be explicitly interpreted as a geometrical realspace projection of the atomic structure around absorbing atoms. The transition from coherent holographic imaging in the reciprocal space to the incoherent imaging in the real space is realized by a continuous increase in the bandwidth of the incident $x$ rays. Therefore, there is no well defined and sharp distinction between the two methods. In a realistic case of a broadband polychromatic x-ray beam, the finite width of the spectrum will produce remnant diffraction fringes [see Fig. 1(c)], which need to be taken into account in the evaluation procedures. However, the signals of individual scatterers strongly localize around interatomic directions and a realspace interpretation is more illustrative.

After the first demonstration of the real-space approach, a tomographic algorithm for $\mathrm{x}$-ray absorption pattern analysis has been proposed. ${ }^{3}$ It analyzes the intensity of bands, corresponding to projections of atomic planes, that are formed due to the superposition of signals from a large number of periodically arranged scatterers as shown in Fig. 1(d). This 


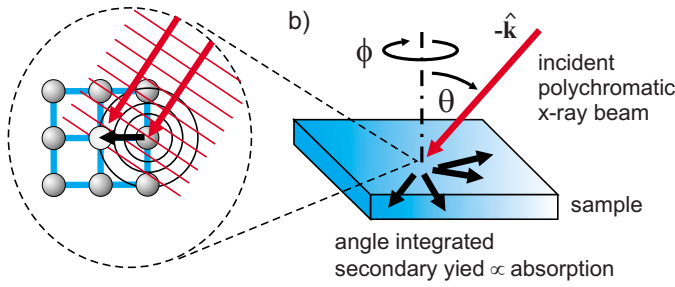

c)

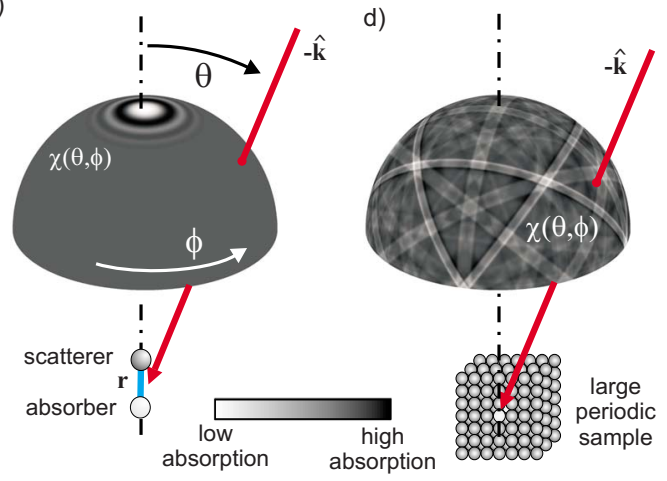

FIG. 1. (Color online) X-ray absorption anisotropy for polychromatic illumination. (a) Interaction between the incident plane wave with spherical waves scattered inside the sample modifies the total $\mathrm{x}$-ray field at the sites of absorbing atoms, which results in anisotropic absorption. (b) Absorption is probed by collecting the secondary yield while the sample is rotated relative to the incidentbeam direction. (c) For a broadband polychromatic illumination, due to a short longitudinal coherence length, the signal of a single scatterer is localized around the interatomic direction. (d) For a periodic sample, the superposition of signals from individual atoms gives rise to a pattern consisting of intensity bands, which can be quantitatively interpreted as projections of the atomic planes. The proposed wavelet approach permits us to detect signals from single atoms, belonging to the local structure of absorbing atoms, in the complex pattern shown in (d). The remnant diffraction fringes visible in (c) make it possible to obtain partial depth information.

technique is capable of determining three-dimensional (3D) crystal structures, however, similar to other recently proposed methods, ${ }^{14,15}$ is insensitive to the geometric arrangement of atoms in the local structure.

In principle, $\mathrm{x}$-ray holographic methods ${ }^{7}$ allow for a full three-dimensional imaging of the averaged local structure around absorbing atoms. However, for monochromatic illumination, x-ray absorption patterns can be obscured by the so-called extinction effects resulting from multiple scattering in the crystal. An in-depth analysis of this effect was presented in Ref. 16. It was shown that the extinction-induced artifacts can interfere with the correct images at the atomic positions in the holographic reconstructions. Extinction effects are minimized when a hard x-ray polychromatic radiation is used to record absorption anisotropy. This is mainly due to the combination of two effects. First, the escape depth of the secondary radiation is in most cases negligible compared to the absorption and extinction lengths of the incident hard $\mathrm{x}$-ray radiation. Second, for polychromatic hard $\mathrm{x}$ rays the scattering takes place in the forward-scattering geometry for which extinction does not influence the secondary yield significantly. ${ }^{16}$ Experimentally the absence of the extinction effects for polychromatic radiation was demonstrated in Ref. 17.

In this paper, we propose a quantitative wavelet transform approach for imaging of the local atomic structure. Contrary to sine and cosine functions, which are used in Fourier transforms, wavelets are well suited for analysis of localized variations in the signal and allow to analyze data at different scales. ${ }^{18}$ All wavelets can be generated from a so-called "mother" wavelet by scaling, translations, or rotations and therefore have the same universal shape. A particular form of the mother wavelet can be adopted for a specific application. In most applications wavelets are oscillatory functions well localized in the real space. The wavelet transform corresponds to a decomposition of the analyzed signal into wavelets, which can be described as a generalized correlation between the signal and the scaled and translated wavelets.

We show, that for a broadband polychromatic illumination, the $\mathrm{x}$-ray absorption anisotropy pattern can be described as a simple linear superposition of wavelet-like functions, each corresponding to a single scatterer. Therefore, the wavelet transform is a natural and an optimal method of analyzing the x-ray absorption anisotropy patterns recorded for polychromatic $x$ rays and imaging of local atomic structure.

The paper is organized as follows. In Sec. II, we show that for a broadband polychromatic x-ray illumination the signals of individual scatterers are localized around interatomic directions. This allows us to use the small-angle approximation and show that all atomic signals have the same universal shape. In, Sec. III we construct a spherical wavelet family, that is, matched to the x-ray absorption anisotropy signals. We demonstrate that there is a direct relationship between the interatomic distance and the wavelet scale, which leads to a depth resolution in reconstructed images. In Sec. IV we demonstrate application of the wavelet filter to $\mathrm{X}$-ray absorption anisotropy data recorded for an InAs crystal. We show that the wavelet filter is capable of providing high-resolution local atomic-structure projections. By changing a single parameter of this filter one is able to obtain projections at different depths. Section V contains conclusions and the appendices present supporting general information about the properties of the two-dimensional and the spherical continuous wavelet transforms.

\section{X-RAY ABSORPTION ANISOTROPY}

\section{A. Arbitrary hard x-ray broadband spectrum}

For monochromatic $\mathrm{x}$ rays, the absorption anisotropy can be written as

$$
\mu(\mathbf{k})=\mu_{a}(k)\left[1+\chi_{0}(\mathbf{k})\right]
$$

where $\mathbf{k}$ is antiparalle ${ }^{19}$ to the wave vector of the incident radiation and $\mu_{a}$ is the absorption coefficient of an isolated atom. ${ }^{20}$ Since $\mathrm{x}$-ray absorption anisotropy $\chi_{0}(\mathbf{k})$ arises from coherent interaction of the incident plane wave $\exp (-i \mathbf{k} \cdot \mathbf{r})$ with spherical waves $\exp (i k r) / r$ scattered inside the sample, it can be written as ${ }^{21}$ 


$$
\chi_{0}(\mathbf{k})=-2 r_{e} \operatorname{Re} \int_{\mathbb{R}^{3}} \rho(\mathbf{r}) \frac{e^{i k r}}{r} e^{-i \mathbf{k} \cdot \mathbf{r}} d^{3} r,
$$

where $r_{e}$ is Thomson scattering length and $\rho(\mathbf{r})$ is the realvalued electron charge distribution inside the sample at position $\mathbf{r}$ relative to absorbing atom. Generalization of Eq. (2) to multiple positions of absorbing atoms is described in Ref. 16.

For polychromatic $\mathrm{x}$ rays the absorption anisotropy becomes

$$
\chi(\hat{\mathbf{k}})=\int_{0}^{\infty} N(k) \chi_{0}(\mathbf{k}) d k,
$$

where $\hat{\mathbf{k}}=\mathbf{k} / k$ and $N(k)$ is the normalized effective wavevector spectrum sensed by the absorbing atoms, which is determined by the spectrum of the incident beam and $\mu_{a}(k) .{ }^{17}$

If the dispersion corrections are negligible, insertion of Eq. (2) into Eq. (3) gives

$$
\chi(\hat{\mathbf{k}})=-2 r_{e} \int_{\mathbb{R}^{3}} \frac{\rho(\mathbf{r})}{r} h(\vartheta, r) d^{3} r .
$$

The signal $h(\vartheta, r)$ is due to a single electron placed at position $\mathbf{r}$ relative to absorbing atom and it is defined as

$$
h(\vartheta, r)=\operatorname{Re} \int_{-\infty}^{\infty} N(k) e^{i k r t} d k,
$$

where

$$
t=1-\cos \vartheta
$$

and $\vartheta=\arccos (\hat{\mathbf{k}} \cdot \hat{\mathbf{r}})$. For any realistic energy spectrum, $N(k)=0$ for $k<0$ and the semi-infinite integral limits in Eq. (3) can be replaced by infinite limits in Eq. (5).

An x-ray absorption experiment requires a reasonably smooth, unimodal, and broadband $N(k)$ spectrum. Let $k_{0}$ be some value characteristic of spectrum $N(k)$, for example, its median, mean, or mode. Thus, $N\left(k+k_{0}\right)$ describes this spectrum centered at zero. Using this shifted wave-vector spectrum, the signal $h(\vartheta, r)$ can be written as

$$
h(\vartheta, r)=\operatorname{Re}\left[e^{i k_{0} r t} n(r t)\right]
$$

where

$$
n(x)=\sqrt{2 \pi} \mathcal{F}\left\{N\left(k+k_{0}\right)\right\}(x)
$$

and the symbol $\mathcal{F}$ denotes a Fourier transform, which is defined as

$$
\mathcal{F}\{N(k)\}(x)=\frac{1}{\sqrt{2 \pi}} \int_{-\infty}^{\infty} N(k) e^{i k x} d k .
$$

The envelope of $h(\vartheta, r)$ is equal to $|n(r t)|$. Since, for a broadband $N(k)$ spectrum, $|n(r t)|$ is well localized around zero, without loss of generality, one can use the small-angle approximation

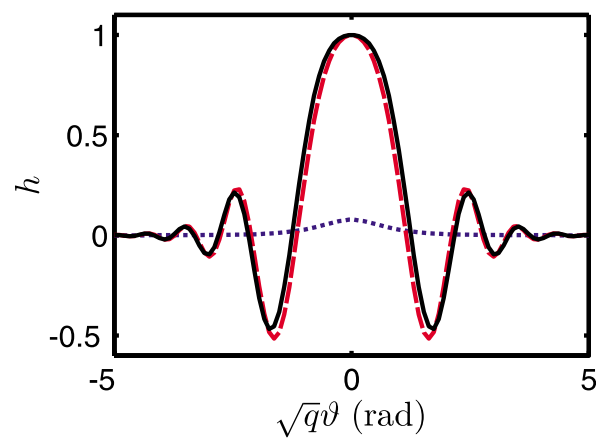

FIG. 2. (Color online) X-ray absorption anisotropy signal $h(\vartheta)$ calculated for a Lorentzian spectrum $N(k)$ with $\beta=\Delta k /\left(2 k_{0}\right)=1 / 4$ (solid line). The dotted curve shows the small difference between signal calculated with and without changing the integration limits in Eq. (5). For comparison, the dashed line shows a wavelet having zero mean, which is discussed in Sec. III. The net anisotropy $\chi$ can be calculated as a simple superposition of functions $h$ multiplied by $-2 r_{e} / r$ factors.

$$
t=1-\cos \vartheta \approx \frac{1}{2} \vartheta^{2}
$$

and write $h(\vartheta, r)$ as

$$
h(\vartheta, r)=\operatorname{Re}\left[\exp \left(\frac{i k_{0} r \vartheta^{2}}{2}\right) n\left(\frac{r \vartheta^{2}}{2}\right)\right] .
$$

Within the small-angle approximations, the functions $h(\vartheta, r)$ are similar, i.e., they have exactly the same shape for all $\mathbf{r}$. The position of the signal on the sphere is determined by $\hat{\mathbf{r}}$ while the scale of $h$ is determined by $r$.

\section{B. Lorentzian spectrum}

In this work, the spectrum $N(k)$ will be assumed to be a Lorentzian curve centered on $k_{0}$ with a full width at half maximum equal to $\Delta k$,

$$
N(k)=\frac{1}{2 \pi} \frac{\Delta k}{\left(k-k_{0}\right)^{2}+(\Delta k / 2)^{2}},
$$

for which it is useful to define an auxiliary quantity

$$
\beta=\frac{\Delta k}{2 k_{0}} .
$$

In principle, this assumption is incompatible with the infinite integration limits in Eq. (5). However for small values of $\beta$ this does not lead to any significant loss of precision (see Fig. 2). For such a spectrum, Eq. (8) becomes

$$
n(x)=e^{-1 / 2 \Delta k|x|}
$$

and the signal $h$ is approximately equal to

$$
h(\vartheta, r) \approx e^{-\beta q \vartheta^{2}} \cos \left(q \vartheta^{2}\right),
$$

where $q=k_{0} r / 2$. The signal $h$, calculated for $\beta=1 / 4$ is plotted in Fig. 2. Figure 3 exemplifies the basic properties of x-ray anisotropy for a broadband $N(k)$ spectrum: localization of individual signals around interatomic directions and their scaling properties. 


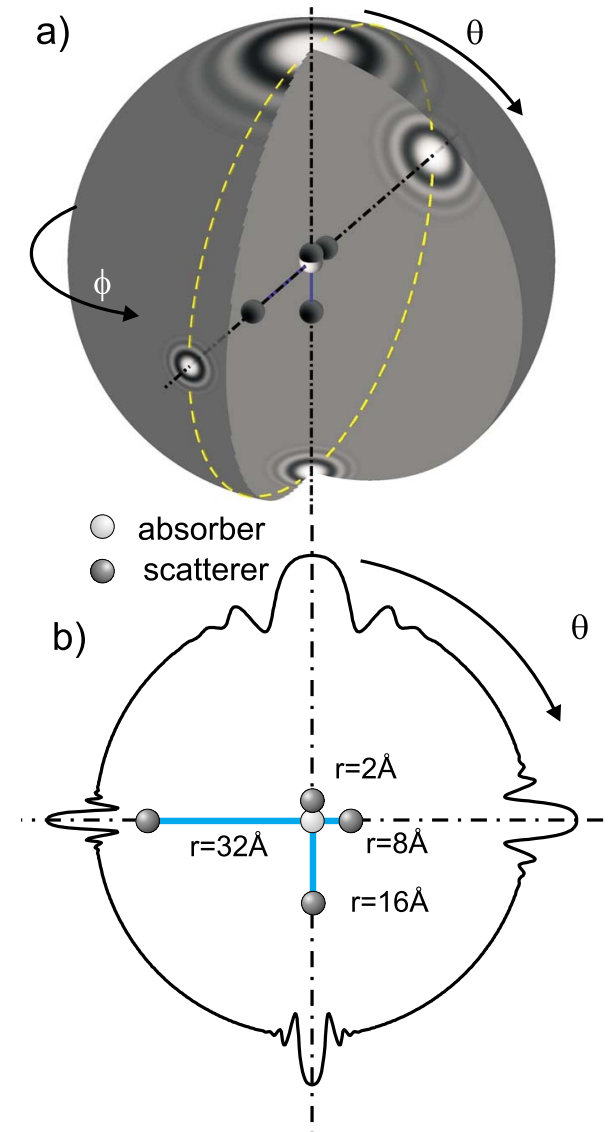

FIG. 3. (Color online) Properties of the absorption anisotropy for polychromatic $\mathrm{x}$ rays: localization around interatomic directions and similarity of signals from scatterers at different positions. (a) Surface plot of signals $h(\theta, r)$ produced by scatterers placed at different positions relative to the absorbing atom. The geometrical arrangement of scatterers is shown in the central part of the plot. All signals have the same shape and are localized around directions connecting a scatterer and an absorber. (b) Polar plot of the same signal calculated at the position depicted by a dashed line in (a). The calculation was performed for $k=50 \AA^{-1}, \beta=1 / 4$, and for interatomic distances $r=(2,8,16,32) \AA$. In order to facilitate a direct comparison of the shape of the signals for different $r$, we did not take into account the $-2 r_{e} / r$ prefactor responsible for the radial decay of the amplitude.

\section{CONTINUOUS WAVELET TRANSFORM}

\section{A. Spherical wavelet transform in small-angle approximation}

Localized and similar functions from Eqs. (11) and (15) are analogous to wavelets. The continuous wavelet transform is extensively used in signal and image processing. ${ }^{22,23} \mathrm{In}$ particular, it has been used in optics ${ }^{24,25}$ and $\mathrm{x}$-ray spectroscopy ${ }^{26}$ for analyzing signals at different scales. For a brief description of the continuous two-dimensional and spherical wavelet transforms see Appendices A and B, respectively.

The continuous spherical wavelet transform, for isotropic wavelets, is defined as a generalized correlation between the analyzed signal and the scaled and rotated wavelets $\psi_{\hat{\mathbf{k}}_{0}, s},{ }^{27}$

$$
\widetilde{\chi}\left(\hat{\mathbf{k}}_{0}, s\right)=\int_{S^{2}} \psi_{\hat{\mathbf{k}}_{0}, s}(\hat{\mathbf{k}}) \chi(\hat{\mathbf{k}}) d \Omega,
$$

where $s>0$ is the scale parameter and $\hat{\mathbf{k}}_{0}$ determines the position of the wavelet on the sphere. The large scales correspond to the slowly varying components of the signal, whereas the smaller scales correspond to finer details. All of the wavelets $\psi_{\hat{\mathbf{k}}_{0}, s}$ can be obtained from a mother wavelet $\psi$ through scaling and rotation. In our approach, the mother wavelet is real valued, localized around the north pole, and isotropic: $\psi(\hat{\mathbf{k}})=\psi(\theta)$, where $\theta$ is the polar angle. The smallangle approximation causes the spherical wavelet transform to become locally equivalent to a two-dimensional wavelet transform. ${ }^{28}$ The mother wavelet is chosen in such a way that for scales $s \leq 1$, the scaling operation can be defined via a strict analogy with the two-dimensional case

$$
\psi_{s}(\theta)=\frac{1}{s} \psi\left(\frac{\theta}{s}\right) .
$$

The shape of the mother wavelet can be adopted for a specific application. The only requirement is that this wavelet meets so-called admissibility condition. For highly localized isotropic spherical wavelets, this condition reduces to a twodimensional zero-mean condition

$$
\int_{0}^{\infty} \psi(\theta) \theta d \theta=0
$$

This criterion, together with localization, implies that a wavelet is an oscillating function. Although the functions in Eq. (15) are localized, similar, and oscillatory, they are not perfectly admissible.

Admissibility can be achieved by adding a small correction term to $h(\vartheta, r)$. We introduce a wavelet family defined as

$$
\psi_{s}(\theta)=\frac{1}{s} e^{-\beta q_{0} \theta^{2} / s^{2}}\left[\cos \left(\frac{q_{0} \theta^{2}}{s^{2}}\right)-\beta \sin \left(\frac{q_{0} \theta^{2}}{s^{2}}\right)\right],
$$

where $q_{0}=k_{0} r_{0} / 2$ and the parameter $r_{0}$ is chosen to ensure the validity of the small-angle approximation for a scale parameters $s \leq 1$. For $\beta=0$, the proposed wavelet $\psi_{s}(\vartheta)$ is perfectly matched to the signal $h(\vartheta, r)$ for scale parameter

$$
s=\left(r / r_{0}\right)^{-1 / 2} \text {. }
$$

The wavelet $\psi_{s}(\theta)$ is compared to the signal $h(\vartheta, r)$ in Fig. 2.

\section{B. Wavelet filter}

Since the wavelets from Eq. (19) are well matched to the signals of individual scatterers, the wavelet transform coefficients will be at a maximum if the wavelet's scale and direction coincide with the position of one of the scatterers. Our simulations show, that the direct use of the transform from Eq. (16) for analyzing absorption anisotropy can be successfully applied to systems consisting of only a few scatterers. For larger systems, it will produce artifacts similar to those observed in single-energy x-ray holography. ${ }^{29}$

Therefore, further analysis is based on the invertibility of the wavelet transform and the real-space interpretation of the 
absorption anisotropy $\chi$. The admissibility criterion guarantees that the wavelet transform is invertible, i.e., that the original signal can be reconstructed from a full set of wavelet coefficients. Consider a filtered linear inversion scheme of the wavelet transform, ${ }^{22,23}$

$$
U(\hat{\mathbf{k}})=C_{\psi} \int_{0}^{\infty} \tilde{\chi}(\hat{\mathbf{k}}, s) w(s) \frac{d s}{s^{2}}
$$

in which the extra window function $w(s)$ is defined as

$$
w(s)=\left\{\begin{array}{cc}
1 & \text { if } s_{c}<s \leq 1 \\
0 & \text { elsewhere }
\end{array}\right.
$$

and $C_{\psi}$ is a constant that only depends on the shape of the wavelet $\psi$. For wavelets defined in Eq. (19)

$$
C_{\psi} \approx 2 k_{0} r_{0} /\left(\pi^{2}-2 \pi \arctan \beta\right) .
$$

Since the integral in Eq. (21) only takes into account a finite range of scales, the function $U(\hat{\mathbf{k}})$ corresponds to a filtered version of $\chi(\hat{\mathbf{k}})$. The upper limit for the scale parameter is set at $s=1$ to ensure the small-angle approximation, whereas the introduction of a cutoff scale $s_{c}$ will remove fine details from the pattern, thereby reducing contributions from distant scatterers. The wavelets $\psi_{\hat{\mathbf{k}}_{0}, s}$ are nonorthogonal and this filter is not perfect, causing the cutoff scale to be fuzzy rather then sharp. The cutoff scale $s_{c}$ and cutoff distance $r_{c}$ are related by

$$
s_{c}=\left(r_{c} / r_{0}\right)^{-1 / 2} \text {. }
$$

The application of the wavelet filter from Eq. (21) to calculated data is demonstrated in Fig. 4. The filter was applied to the simple signal from Fig. 3. Application of the wavelet filter to such a simple test pattern does not provide any new information. However, in the next section we will apply the wavelet filter to a pattern recorded for an almost-perfect crystal. For a crystal, the superposition of signals from periodically arranged atoms masks the signals coming from the nearest atoms. We will show that the wavelet filter allows one to suppress the contribution from distant atoms and to reveal projections of the local structure around absorbing atoms.

\section{ANALYSIS OF X-RAY ABSORPTION ANISOTROPY RECORDED FOR InAs(001) CRYSTAL}

\section{A. Experiment and data analysis}

In this section, we apply the proposed wavelet formalism to the X-ray absorption anisotropy data recorded for an InAs crystal with a (001) orientation. InAs has the zinc-blende structure (space group $F \overline{4} 3 m$ ) and the lattice constant $a_{0}$ $=6.0583 \AA$. The X-ray absorption anisotropy pattern was measured at HASYLAB on the beamline $\mathrm{C} 1$. In order to obtain a broadband spectrum $N(k)$, the beam from a bending magnet passed through a 15 -mm-thick $\mathrm{Al}$ absorber and was limited by slits to a size of $0.3 \times 0.3 \mathrm{~mm}^{2}$. The beam intensity was monitored using a $\mathrm{Si}$ photodiode placed directly in the beam path. The sample absorption was probed by measuring the total electron yield with a compact gas-filled de-

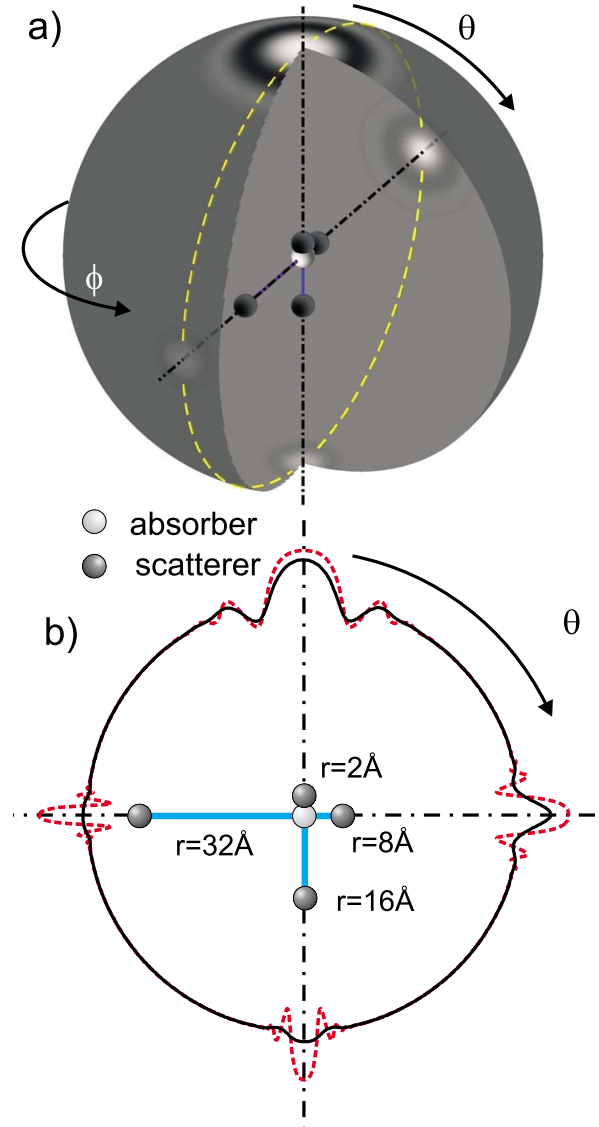

FIG. 4. (Color online) The wavelet filter applied to the data from Fig. 3. (a) Surface plot. (b) Polar plot. The dashed line shows the original signal. The cutoff distance $r_{c}$ was set to $10 \AA$ whereas the parameter $r_{0}$ was set to $1 \AA$. While the signals from the distant scatterers are strongly suppressed, the forward-scattering features of near scatterers are still intense and can be used for a real-space determination of their positions. The change in the cutoff scale of the wavelet filter allows for a depth resolution.

tector operating in a current mode. The sample was rotated around two axes relative to the direction of the incident beam. The total acquisition time was $\sim 24 \mathrm{~h}$. The details of the experimental setup are described in Ref. 17. The absorption anisotropy was obtained from raw data by background subtraction and using symmetrization operations from the $F \overline{4} 3 m$ space group. The background was subtracted separately for each azimuthal scan using smoothing splines. ${ }^{30}$

Since a direct measurement of the effective spectrum $N(k)$ is cumbersome, the most important parameters of this spectrum were obtained directly from the experimental pattern. Figure 5(a) shows a profile of the x-ray absorption anisotropy recorded in the vicinity of an intensity band corresponding to the projection of the $(1 \overline{1} \overline{1})$ plane. The parameters of the Lorentzian $N(k)$ spectrum were obtained from this data by a fit of a theoretical curve, according to the formalism introduced in Ref. 17. The parameters obtained in such a procedure, $k_{0}=(22.2 \pm 0.3) \AA^{-1}\left(E_{0} \approx 44.4 \mathrm{keV}\right)$ and $\Delta k=(10.7 \pm 2.1) \AA^{-1}(\Delta E \approx 21.4 \mathrm{keV})$, were used to construct the wavelet family and for simulation of the x-ray absorption pattern. In order to estimate the deviation of the 
(a)

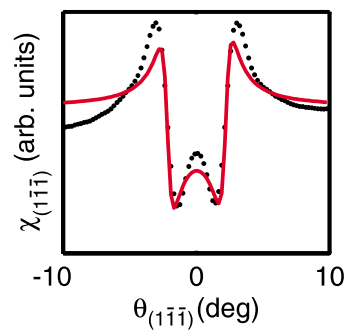

(b)

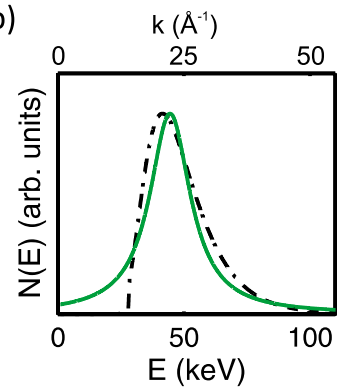

FIG. 5. (Color online) Determination of the parameters of the effective spectrum $N(k)$. (a) A profile of the recorded x-ray absorption anisotropy in the vicinity of an intensity band corresponding to the projection of the $(1 \overline{1} \overline{1})$ plane (points). The parameters of the Lorentzian $N(k)$ spectrum were obtained by a fit of a theoretical curve (solid line). (b) The Lorentzian spectrum $N(k)$ calculated for parameters obtained from fit (a) is shown as a solid line. The dashdot line shows the spectrum calculated using known parameters of the beamline and sample.

Lorentzian spectrum from the experimental one, we also independently calculated the spectrum $N(k)$ using the synchrotron source emission characteristics, transmission of all elements placed in the beam and the energy dependence of the absorption in the sample. The calculated spectrum is compared to the Lorentzian spectrum in Fig. 5(b). Though the agreement is not perfect, such precision is sufficient for a quantitative analysis, as will be shown in the next subsection by a comparison between recorded and simulated data.

The x-ray projections were obtained from x-ray anisotropy data using the fast spherical wavelet transform procedure in the YAW toolbox ${ }^{27,31}$ in the MATLAB computing environment. Since $U(\hat{\mathbf{k}})$ is a filtered projection of $-\rho(\mathbf{r})<0$, it is convenient to present data using a modified function $U_{0}(\hat{\mathbf{k}})$ that is equal to $U(\hat{\mathbf{k}})$ for negative values and zero otherwise. To ensure that the small-angle approximation is valid, $r_{0}$ was set to $2 \AA$ while the cutoff distance $r_{c}$, defined in Eq. (24), was varied to obtain depth information.

\section{B. Results and discussion}

Figure 6(a) shows the x-ray absorption anisotropy pattern recorded for our InAs sample. The most visible features are bands corresponding to the projections of atomic planes. For comparison, Fig. 6(b) shows x-ray anisotropy calculated using the formalism described in Ref. 17. For the calculation input, we only used two nonstructural parameters $k_{0}$ and $\Delta k$ of the Lorenztian-shaped $N(k)$ spectrum, which were directly obtained from the experimental data. The calculation assumes $\sim 1 / 4$ ratio of As-to-In absorption. Thus, this pattern is a linear combination of patterns corresponding to In and As absorbing atoms. Apart from the contrast, the agreement between theory and experiment is excellent. This confirms the real-space character of the $\mathrm{x}$-ray absorption anisotropy patterns and the lack of so-called extinction effects. The decrease in the contrast of the experimental pattern is due to the detector background signal and the presence of a thin amor- (a) experiment

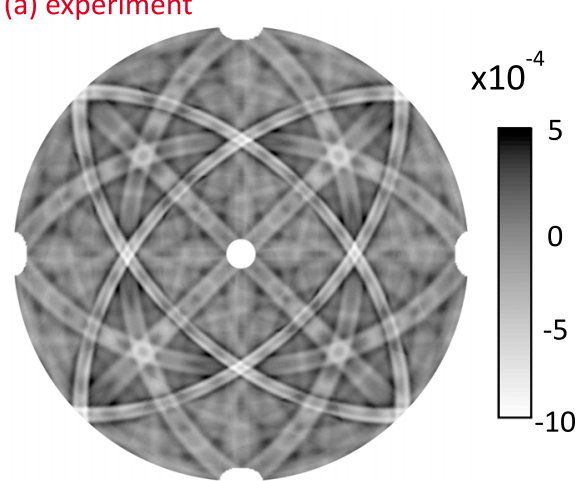

(b) calculation

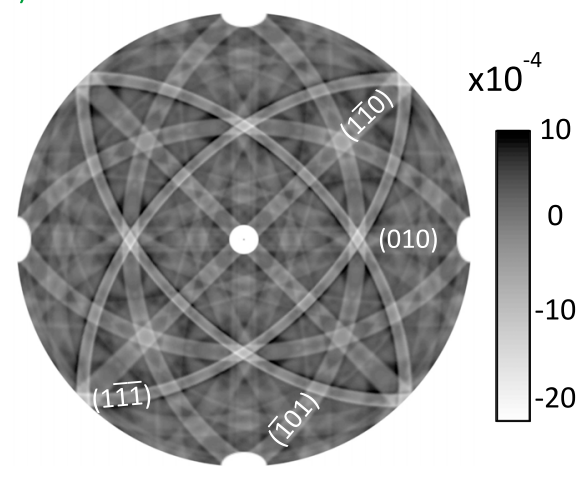

FIG. 6. (Color online) X-ray absorption anisotropy signals for an InAs(001) crystal. (a) Experimental data. (b) Data calculated using kinematical theory for: $k_{0}=22.2 \AA^{-1}\left(E_{0} \approx 44.4 \mathrm{keV}\right)$ and $\Delta k=10.7 \AA^{-1}(\Delta E \approx 21.4 \mathrm{keV})$. The patterns are presented as fisheye projections of the complete hemisphere. Part of the data where the background subtraction was ambiguous was set to zero.

phous layer at the sample surface which does not contribute to the anisotropy.

The wavelet analysis of the experimental data is demonstrated in Fig. 7. X-ray projection of the local structure in InAs, obtained with a wavelet filter from the experimental $\mathrm{x}$-ray absorption anisotropy pattern is shown in Fig. 7(b). For comparison, Fig. 7(a) presents a geometrical fish-eye view of a small InAs cluster. The fish-eye image was calculated with a ray-tracing software (POV-RAY) for a small cluster (30 ̊ radius) of spheres, arranged in the InAs structure, using the principles of geometrical optics. The observation point was placed at the position of the central In atom and a fish-eye perspective was used. In the resulting image, In and As atoms are shown as dark and bright spheres, respectively. Their dimensions are proportional to the atomic number and inversely proportional to the distance from the central atom. For clarity, atoms at distances larger than $10 \AA$ are shown with decreased intensity. Exactly the same fish-eye projection $(x=\theta \cos \phi$ and $y=\theta \sin \phi)$ was used for the presentation of the experimental x-ray angular maps.

In addition, Fig. 7(c) shows the result of the wavelet filter applied to the pattern calculated for a small InAs cluster with a radius of $10 \AA$. The atoms included in this calculation are shown with enhanced intensity in panel (a). The data calculated in (c) correspond to a weighted linear superposition of the projections around the In and As atoms. From the view point of an As atom, the fish-eye view from (a) is rotated by 
(a)

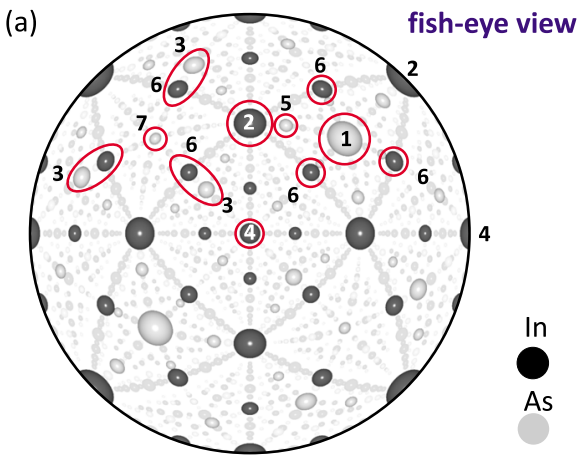

(b)

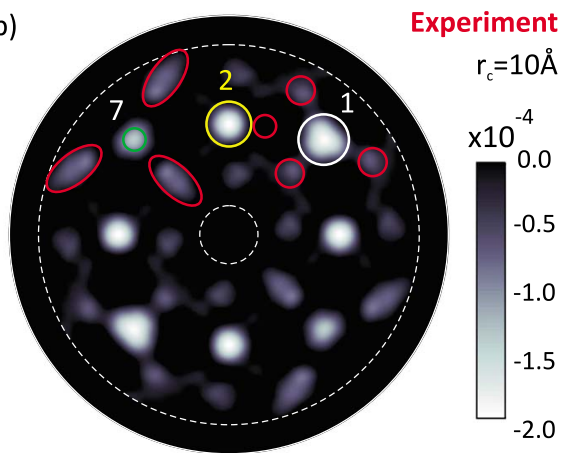

(c)

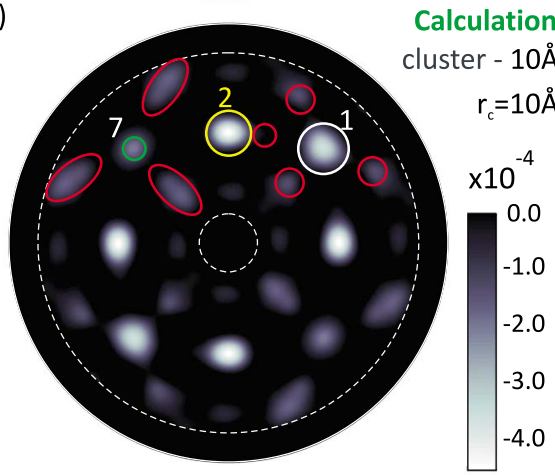

FIG. 7. (Color online) Projections of the local structure in InAs. (a) Fish-eye view of a small InAs cluster (observation point is placed at an In atom site). The sizes of the balls are proportional to the atomic number and inversely proportional to the distance from the central atom. The oval shapes mark the strongest scatterers. The labels correspond to coordination shell number. (b) Experimental x-ray projection $U_{0}$ obtained from the data shown in Fig. 6(a) using a wavelet filter. The areas, where the edge effects are important, were set to zero. (c) X-ray projection $U_{0}$ obtained from the pattern calculated for a small InAs cluster with $10 \AA$ radius. The angular and intensity scales are identical to those in Fig. 6.

$90^{\circ}$ and the positions of In and As are interchanged.

The $\mathrm{x}$-ray projections obtained from the experimental and calculated data are in excellent agreement. A comparison of the experimental data with the fish-eye view indicates that the projections of nearest neighbors and next nearest neighbors are clearly resolved. More distant atoms are still visible but their projections overlap due to a finite angular resolution. The size of atomic projections decreases with their distance from the central atoms, similarly as in the fish-eye view. Remarkably, the nonspherical shape of the atomic projections has a clear real-space interpretation. This shape is connected to the local environments of scattering atoms. The
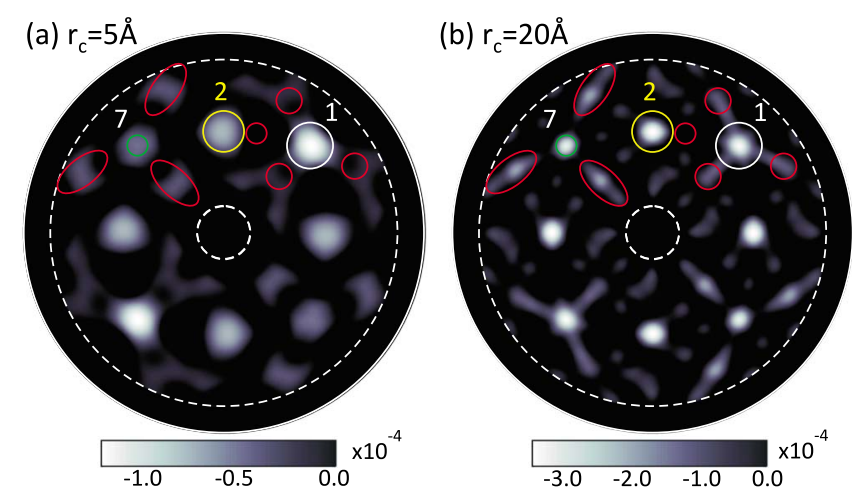

FIG. 8. (Color online) Beyond projection imaging: depth resolution. Experimental x-ray projections $U_{0}$ obtained using a wavelet filter for different cutoff distances (a) $r_{c}=5 \AA$ and (b) $r_{c}=20 \AA$. In (a) the most intense spots correspond to the projections of nearest neighbors. In Fig. 7(b), the spots of nearest neighbors and next nearest neighbors have comparable intensities. In (b), the spots corresponding to more distant atoms become stronger.

sensitivity of the method to the local structure is exemplified by different intensities at the spots labeled 1 and 7 . The corresponding crystal directions [ $\overline{1} \overline{1} 1]$ and [ $\overline{1} 11]$ have the same linear electron density. Thus, the visible difference is due to the different distances to nearest atoms lying on these directions. Due to the ability of the wavelet transform to analyze localized variation in the signal, the missing data in the pattern did not influence the projections of the local structure.

For a constant value $r_{c}$ of the wavelet filter, the visibility of atomic images is mainly noise limited. The x-ray anisotropy images allow us to observe the projections of individual In atoms $(Z=49)$ up to the sixth coordination sphere $(r=7.49 \AA)$ and As atoms $(Z=33)$ up to the third coordination sphere $(r=5.02 \AA)$. For both cases, the ratio $2 Z r_{e} / r$ is approximately equal to $3.7 \times 10^{-4}$. This number, which can be directly related to the observed anisotropy, can be taken as a quantitative measure of the achieved sensitivity.

Figure 8 demonstrates another benefit of using the wavelet formalism for analyzing absorption anisotropy: by changing the cutoff distance $r_{c}$ of the wavelet filter, one can obtain depth information. Although the radial resolution is sufficient for assigning nearby atoms to coordination spheres, it is insufficient for detecting small changes in the interatomic distances.

\section{Resolution and the choice of the effective $N(k)$ spectrum}

In this subsection we present a short discussion concerning the resolution and the proper choice of the effective spectrum $N(k)$ for future applications. For a Lorentzian spectrum $N(k)$, the angular resolution can be related to the width of the central maximum of the function $h$ from Eq. (15), or more precisely to the angular distance between its first zeros

$$
\Delta \theta=2 \sqrt{\pi /\left(k_{0} r\right)} .
$$

In practice, the increase in $k_{0}$ can be realized by increasing the thickness of an absorber placed in the x-ray beam, which shifts the spectrum toward higher $k$ values. 
The wavelet analysis is only possible for highly localized signals, i.e., when the small-angle condition from Eq. (10) is fulfilled. The localization of the signals around interatomic directions is determined by the ratio $\Delta k r$, which determines the shape of the envelope of the signal $h$. For example, for $\Delta k r=25$, the envelope of the signal drops to $1 / e^{2}$ of its maximum value at an angle $\sim 33^{\circ}$, where the small-angle approximation of Eq. (10) has a relative error in the range of $3 \%$. Thus, for the nearest-neighbor distance $r=2.5 \AA$, the bandwidth should be $\Delta k \gtrsim 10 \AA^{-1}$ (or equivalently $\Delta E$ $\gtrsim 20 \mathrm{keV}$ ).

The localization of the signal around interatomic direction is accompanied by the smearing of the higher-order diffraction fringes. This deteriorates the resolution along radial direction. The radial resolution, calculated by a direct insertion of Eqs. (15) and (19) into Eq. (16) can be approximated as ${ }^{32}$

$$
\Delta r=4 r \beta,
$$

where the parameter $\beta$ was defined in Eq. (13). This value determines the sharpness of the cutoff value $r_{c}$ of the wavelet filter. Thus, images of atoms located at $r \lesssim r_{c}-2 r \beta$ are weakly influenced by the filter, whereas the intensities of more distant images, apart from the obvious $1 / r$ factor, are strongly suppressed.

The presented method requires a reasonably smooth energy spectrum. Any absorption edge present at an energy where the spectrum $N(k)$ has significant values, will produce a discontinuity in the effective energy spectrum and, via Fourier transform, will strongly influence the shape of $h$ function [c.f. Eqs. (7) and (8)]. Thus, there exists an upper limit for absorption edges energy of elements inside the sample. Since a realistic spectrum usually has a positive skewness and drops quite rapidly to zero at the lower-energy side [see. Fig. 5 and Refs. 2 and 17], it is sufficient if the highest absorption edge is lower than $E_{0}-\Delta E / 2$. For example, for an effective spectrum with $E_{0}=50 \mathrm{keV}$ and $\Delta E_{0}=25 \mathrm{keV}$, all absorption edges should be at energies $E_{a} \lesssim 37.5 \mathrm{keV}$. In addition, this condition ensures that the dispersion corrections, which are significant near absorption edges, are negligible.

Concluding, the increase in $\Delta k$ improves the radial resolution but simultaneously worsens the accuracy of the smallangle approximation. Thus, a value between $\Delta k=5 \AA^{-1}$ and $\Delta k=15 \AA^{-1}(10$ and $30 \mathrm{keV})$ seems to be a reasonable choice. For a constant $\Delta k$, the increase in $k_{0}$ improves both the radial and angular resolutions and makes it possible to study samples containing elements with higher atomic number.

\section{CONCLUSIONS}

In summary, we have shown that for a broadband polychromatic illumination x-ray absorption anisotropy can be calculated as a linear superposition of localized wavelet-like signals corresponding to individual scatterers. The wavelet transform is the natural method for analyzing such data and allows to obtain a direct information about the local structure of absorbing atoms. Its application to experimental data allowed to obtain high-resolution projections of the local structure at different depths. The depth or radial resolution is lim- ited as compared to other methods, ${ }^{7,33}$ however this limitation is compensated by the robust character of the realspace approach, which overcomes the inherent problems of $\mathrm{x}$-ray holography associated with long-range order and multiple-scattering effects. ${ }^{16,29}$ In addition, the localized character of wavelets makes the method insensitive to missing data.

In this paper, we applied the wavelet formalism for imaging the local atomic structure from x-ray absorption anisotropy, which was recorded for an almost-perfect crystal. As far as the imaging of local structure is concerned, the presence of a perfect long-range order is a problem rather than an advantage. The approach should also work for nonperfect crystals, thin films, and buried layers. The presented method provides information that is complementary to X-ray absorption fine structure, which is a powerful tool for the determination of local interatomic distances. However, in order to efficiently apply the proposed method to chemically resolved $\mathrm{x}$-ray imaging, there must be experimental progress made on the detection of characteristic radiation.

The wavelet approach could also be applied to the analysis of neutron holograms ${ }^{33}$ recorded with polyenergetic beams. Further research will determine if the wavelet technique, despite multiple scattering, is capable of analyzing electron-diffraction data, ${ }^{34-37}$ e.g., filtering out images of individual atomic strings ${ }^{38}$ observed in simulated electrondiffraction patterns.

\section{ACKNOWLEDGMENTS}

This work was supported by Polish Ministry of Science and Higher Education (Grant No. N202 012 32/0628). The access to synchrotron was supported by DESY and the European Community [Contract No. RII3-CT-2004-506008 (IA-SFS)].

\section{APPENDIX A: TWO-DIMENSIONAL WAVELETS}

A two-dimensional function $\psi(\mathbf{x})$ can be called a wavelet if it satisfied the so-called admissibility condition. For square-integrable functions, this condition reduces to a zeromean condition, ${ }^{22,23}$

$$
\int_{\mathbb{R}^{2}} \psi(\mathbf{x}) d^{2} x=0 .
$$

From a wavelet $\psi$ one generates shifted, rotated, and scaled wavelets. In this work we discuss isotropic real-valued wavelets. In this case only shifted and scaled wavelets are generated,

$$
\psi_{\mathbf{x}_{0}, s}(\mathbf{x})=\frac{1}{s} \psi\left(\frac{\mathbf{x}-\mathbf{x}_{0}}{s}\right) .
$$

The continuous wavelet transform of a function $f(\mathbf{x})$ is defined as

$$
\tilde{f}\left(\mathbf{x}_{0}, s\right)=\int_{\mathbb{R}^{2}} \psi_{\mathbf{x}_{0}, s}(\mathbf{x}) f(\mathbf{x}) d^{2} x .
$$

The wavelet coefficient $\tilde{f}\left(\mathbf{x}_{0}, s\right)$ is a measure of the correlation of the analyzed function $f(\mathbf{x})$ with the wavelet $\psi_{\mathbf{x}_{0}, s}(\mathbf{x})$. 
This correlation is calculated at different scales. Usually, the wavelet transform is applied to discrete data. The term continuous means that both the scale parameter $s$ and the translation parameter $\mathbf{x}_{\mathbf{0}}$ are changing continuously.

The admissibility condition ensures that there exists an inverse transform. Since $\psi_{\mathbf{x}_{0}, s}(\mathbf{x})$ are nonorthogonal the information contained in the wavelet coefficients $\tilde{f}\left(\mathbf{x}_{0}, s\right)$ is redundant. However, because of this redundancy, there is more than one reconstruction formula. For example, a simple integral over wavelength scales

$$
f(\mathbf{x})=C_{\psi} \int_{0}^{\infty} \tilde{f}(\mathbf{x}, s) \frac{d s}{s^{2}}
$$

reconstructs the original signal. The constant $C_{\psi}$ depends only on the shape of the mother wavelet $\psi$.

\section{APPENDIX B: GENUINE SPHERICAL WAVELET TRANSFORM}

Extension of the wavelet transform to a spherical surface is nontrivial. Since the sphere is compact, scaling is difficult to define. An elegant solution for constructing wavelets on a sphere is based on the stereographic projection, which maps the sphere onto the tangent plane at the north pole. ${ }^{27,28} \mathrm{~A}$ point $\hat{\mathbf{k}}$ on the sphere $S^{2}$, having spherical coordinates $(\theta, \phi)$, is projected onto a point in the tangential plane with polar coordinates $[\varrho(\theta), \phi]$, where $\varrho(\theta)=2 \tan (\theta / 2)$. This projection induces a unitary mapping $\Pi$ of square-integrable functions on the sphere to square-integrable functions on $\mathbb{R}^{2} \mathrm{de}$ fined by

$$
\Pi \psi(\rho, \phi)=\left(1+\frac{\rho^{2}}{4}\right)^{-1} \psi\left(2 \arctan \frac{\rho}{2}, \phi\right) .
$$

By means of $\Pi$, one defines the admissibility condition on the sphere: the wavelet $\psi(\theta, \phi)$ is admissible, if $\Pi \psi$ fulfills the admissibility condition Eq. (A1). Thus, for isotropic wavelets $\psi(\hat{\mathbf{k}})=\psi(\theta)$, the admissibility condition reads

$$
\int_{0}^{\pi} \psi(\theta) \frac{\sin (\theta)}{1+\cos (\theta)} d \theta=0 .
$$

Since $\Pi$ can be inverted, the scaling of spherical wavelets can be done by first projecting the wavelet to the plane, scaling the planar wavelet and then back projecting the scaled wavelet: $\psi_{s}=\Pi^{-1}\left[(\Pi \psi)_{s}\right]$.

Thus, for spherical wavelets, the scaling operation is defined as

$$
\psi_{s}(\theta)=\lambda(\theta, s)^{1 / 2} \psi\left(\theta_{s}\right),
$$

where $\tan \left(\theta_{s} / 2\right)=s^{-1} \tan (\theta / 2)$ and the prefactor

$$
\lambda(\theta, s)=\frac{4 s^{2}}{\left(s^{2}-1\right) \cos \theta+s^{2}+1}
$$

assures conservation of the $L^{2}$ norm.

The motion of the wavelets is accomplished by a direct rotation on the sphere. Rotation of the mother wavelet from the north pole to a point $\hat{\mathbf{k}}_{0}=\left(\theta_{0}, \phi_{0}\right)$ is implemented by the inverse rotation $R^{-1}\left(\hat{\mathbf{k}}_{0}\right)$ acting on the spherical coordinates

$$
\psi_{\mathbf{k}_{0}, s}(\hat{\mathbf{k}})=\psi_{s}\left[R^{-1}\left(\hat{\mathbf{k}}_{0}\right) \hat{\mathbf{k}}\right]
$$

where the rotation matrix $R\left(\hat{\mathbf{k}}_{0}\right)$ is defined as: $R\left(\hat{\mathbf{k}}_{0}\right)$ $=R_{\hat{\mathbf{z}}}\left(\phi_{0}\right) R_{\hat{\mathbf{y}}}\left(\theta_{0}\right)$. Now, the continuous spherical wavelet transform can be defined accordingly to Eq. (16).

In this work, we use the small-angle approximation for the adaptation of the spherical wavelet transform to analyze $\mathrm{x}$-ray absorption anisotropy. Therefore, all expressions are approximated up to second order of $\theta$. This corresponds to an approximation of Eq. (10). The angular factor of the admissibility condition of Eq. (B2) is approximated as $\sin \theta /(1$ $+\cos \theta) \approx \theta / 2$. Similarly, the factor present in the spherical correlation of function $f(\hat{\mathbf{k}})$ with scaled wavelets from Eq. (16) is approximated as $\lambda(\theta, s)^{1 / 2} \sin \theta \approx \theta / s$ and $\tan (\theta / 2)$ $\approx \theta / 2$. In such an approximation, the admissibility criterion for isotropic and localized wavelets is given by Eq. (18), and, for sufficiently small scales, the scaling operation reduces to Eq. (17).

\footnotetext{
*pawel.korecki@uj.edu.pl

${ }^{1}$ S. G. Benka and G. B. Lubkin, Phys. Today 48 (11), 23 (1995).

${ }^{2}$ P. Korecki and G. Materlik, Phys. Rev. Lett. 86, 2333 (2001).

${ }^{3}$ P. Korecki, M. Tolkiehn, D. V. Novikov, G. Materlik, and M. Szymonski, Phys. Rev. Lett. 96, 035502 (2006).

${ }^{4}$ T. Gog, P. M. Len, G. Materlik, D. Bahr, C. S. Fadley, and C. Sanchez-Hanke, Phys. Rev. Lett. 76, 3132 (1996).

${ }^{5}$ K. Hayashi, M. Matsui, Y. Awakura, T. Kaneyoshi, H. Tanida, and M. Ishii, Phys. Rev. B 63, 041201(R) (2001).

${ }^{6}$ M. Kopecky, A. Lausi, E. Busetto, J. Kub, and A. Savoia, Phys. Rev. Lett. 88, 185503 (2002).

${ }^{7}$ G. Faigel, G. Bortel, C. Fadley, A. Simionovici, and M. Tegze, X-Ray Spectrom. 36, 3 (2007).

${ }^{8}$ K. Hayashi, T. Hayashi, T. Shishido, E. Matsubara, H. Makino, T. Yao, and T. Matsushita, Phys. Rev. B 76, 014119 (2007).
}

${ }^{9}$ J. J. Barton, Phys. Rev. Lett. 61, 1356 (1988).

${ }^{10}$ J. J. Barton, Phys. Rev. Lett. 67, 3106 (1991).

${ }^{11}$ Y. Nishino, J. Miao, and T. Ishikawa, Phys. Rev. B 68, 220101(R) (2003).

${ }^{12}$ Y. Nishino, Y. Takahashi, N. Imamoto, T. Ishikawa, and K. Maeshima, Phys. Rev. Lett. 102, 018101 (2009).

${ }^{13}$ M. Tegze, G. Faigel, S. Marchesini, M. Belakhovsky, and A. I. Chumakov, Phys. Rev. Lett. 82, 4847 (1999).

${ }^{14}$ S. Marchesini et al., Phys. Rev. B 66, 094111 (2002).

${ }^{15}$ T. Matsushita, F. Z. Guo, M. Suzuki, F. Matsui, H. Daimon, and K. Hayashi, Phys. Rev. B 78, 144111 (2008).

${ }^{16}$ P. Korecki, D. V. Novikov, M. Tolkiehn, and G. Materlik, Phys. Rev. B 69, 184103 (2004).

${ }^{17}$ P. Korecki, M. Tolkiehn, D. V. Novikov, G. Materlik, and M. Szymonski, Phys. Rev. B 74, 184116 (2006). 
${ }^{18}$ Wavelets: A Tutorial in Theory and Applications, edited by C. K. Chui (Academic, New York, 1992).

${ }^{19}$ In previous works we used a different convention, in which the vector $\mathbf{k}$ was parallel to the direction of the incident beam. The present convention allows us to write subsequent equations in a compact form. Note, that all results presented in this work are independent of the particular definition of $\mathbf{k}$.

${ }^{20}$ Y. Nishino and G. Materlik, Phys. Rev. B 60, 15074 (1999).

${ }^{21}$ B. Adams, D. V. Novikov, T. Hiort, G. Materlik, and E. Kossel, Phys. Rev. B 57, 7526 (1998).

${ }^{22}$ M. Farge, Annu. Rev. Fluid Mech. 24, 395 (1992).

${ }^{23}$ J.-P. Antoine, CWI Q. 11, 323 (1998).

${ }^{24}$ L. Onural, Opt. Lett. 18, 846 (1993).

${ }^{25}$ C. Buraga-Lefebvre, S. Cotmellec, D. Lebrun, and C. Ozkul, Opt. Lasers Eng. 33, 409 (2000).

${ }^{26}$ H. Funke, A. C. Scheinost, and M. Chukalina, Phys. Rev. B 71, 094110 (2005).

${ }^{27}$ J.-P. Antoine, L. Demanet, L. Jacques, and P. Vandergheynst, Appl. Comput. Harmon. Anal. 13, 177 (2002).

${ }^{28}$ Y. Wiaux, L. Jacques, and P. Vandergheynst, Astrophys. J. 632,
15 (2005).

${ }^{29}$ S. S. Fanchenko, M. Tolkiehn, D. V. Novikov, A. Schley, and G. Materlik, Phys. Rev. B 70, 106102 (2004).

${ }^{30}$ C. de Boor, A Practical Guide to Splines, Applied Math. Sciences Vol. 27 (Springer-Verlag, New York, 2001).

${ }^{31}$ L. Jacques, A. Coron, P. Vandergheynst, and A. Rivoldini, The YAWTb toolbox: Yet Another Wavelet Toolbox, http:// rhea.tele.ucl.ac.be/yawtb

${ }^{32}$ D. K. Saldin, G. R. Harp, B. L. Chen, and B. P. Tonner, Phys. Rev. B 44, 2480 (1991).

${ }^{33}$ L. Cser, G. Krexner, M. Marko, I. Sharkov, and G. Torok, Phys. Rev. Lett. 97, 255501 (2006).

${ }^{34}$ J. Osterwalder, T. Greber, A. Stuck, and L. Schlapbach, Phys. Rev. B 44, 13764 (1991).

${ }^{35}$ C. S. Fadley, Surf. Sci. Rep. 19, 231 (1993).

${ }^{36}$ M. Seelmann-Eggebert, R. Fasel, E. C. Larkins, and J. Osterwalder, Phys. Rev. B 48, 11838 (1993).

${ }^{37}$ A. Winkelmann, C. S. Fadley, and F. J. G. de Abajo, New J. Phys. 10, 113002 (2008).

${ }^{38}$ J. C. H. Spence and C. Koch, Phys. Rev. Lett. 86, 5510 (2001). 